\title{
Investigation of Superluminal Motion of Free Spin-half Particles in Spacetime
}

\author{
Emmanuel Gazoya ${ }^{1,2}$, Francis Oduro ${ }^{2} \&$ Edward Prempeh $^{2}$ \\ ${ }^{1}$ National Nuclear Research Institute / ARC, Ghana Atomic Energy Commission, Accra, Ghana \\ ${ }^{2}$ Department of Mathematics, Kwame Nkrumah University of Science \& Technology, Kumasi, Ghana \\ Correspondence: Emmanuel Gazoya, National Nuclear Research Institute / ARC, Ghana Atomic Energy Commission, \\ Accra, Box LG 80, Ghana. Tel: 233-271-017-044. E-mail: gazoya2001@yahoo.com
}

\author{
Received: August 11, 2015 Accepted: September 1, 2015 Online Published: October 16, 2015 \\ doi:10.5539/jmr.v7n4p12 URL: http://dx.doi.org/10.5539/jmr.v7n4p12
}

\begin{abstract}
The possibility of free spin-1/2 particles (also called Dirac particles) superluminal motion in spacetime, is investigated. The universal cover of the entire Lorentz group $\mathcal{L}$ consists of $S L(2, \mathbb{C})$ and the spinor map so that to obtain a relativistically invariant description of the state of an electron, one looks to the representations of $S L(2, \mathbb{C})$, that is, to the 2 -valued representation of $\mathcal{L}$, known as spinors. We restrict our approach to realistic one-particle systems along with the "positive energy" and utilize the free Dirac waves propagating in the $z$-direction. The Dirac wave function $\psi(x, t)$ is considered as a "classical field" and the corresponding wave equation is derived from a Lagrange function. Using the symmetrized Dirac Lagrange density, it is observed that variation in spin angular momentum (in the light cone) leads to causality violation, whereas variation in orbital angular momentum does not. Consequently, it is shown that the expectation value of the relative translational velocity component of the spinning free electron exceeds light speed.
\end{abstract}

Keywords: free spin-half particles, free Dirac particles, Dirac spinors, superluminal motion, relativistic wave equation, spin angular momentum, orbital angular momenta, symmetrized Dirac Lagrange density, Lorentz boost, causality violation.

\section{Introduction}

The universal existential problem of particles capable of superluminal motion has been of great interest at the heart of many controversies and occupied a lot of authors' attention in mathematical physics, for decades. Though special relativity is not in conflict with faster-than-light phenomena, many regard it paradoxical the fact that the speed of relativistic particles always averages light velocity. No one can deny that the demonstration of superluminal particles existence underpins the net advance of science. In this paper, we go beyond the usual luminal aspect of free fermions (as shown somewhere in other literature, where the unsymmetrized Dirac Lagrange density is used) to prove these particles are superluminal, employing the symmetrized Lagrangian.

To obtain a high resolution probe, high energies and momenta are requisite. So, the covariant generalization of the Shrödinger equation becomes a necessity. We leave the theory of the principles of special relativity and Lorentz transformations, and their application to the construction of special relativistic wave equations for fermions and bosons, the Dirac and Klein-Gordon equations, to the literature: Greiner (1994, 2000); Greiner \& Reinhardt (1996); Naber (1992); Guidry (1991); Carmeli (2000), and references therein.

The study of one-body problem necessarily passes by the review of classical field theory, see Greiner \& Reinhardt (1996). One must reckon with the important statement of Noether's theorem by which each continuous symmetry transformation leads to a conservation law. The conserved quantity $G:=\int_{V} d^{3} x f_{0}(x)$ is defined from the Lagrange density through the conserved Noether's current given by

$$
f_{\mu}(x)=\frac{\partial \mathcal{L}(x)}{\partial\left(\partial^{\mu} \phi_{r}\right)} \delta \phi_{r}(x)-\left[\frac{\partial \mathcal{L}(x)}{\partial\left(\partial^{\mu} \phi_{r}\right)} \frac{\partial \phi_{r}}{\partial x^{v}}-g_{\mu \nu} \mathcal{L}(x)\right] \delta x^{v},
$$

A first application of Noether's theorem concerns invariance under translation and leads to the conserved canonical energymomentum tensor: $\Theta_{\mu \nu}=\frac{\partial \mathcal{L}(x)}{\partial\left(\partial^{\mu} \phi_{r}\right)} \frac{\partial \phi_{r}}{\partial x^{\nu}}-g_{\mu \nu} \mathcal{L}$. This implies four conserved quantities, which are the energy $E$ and the momentum vector $\boldsymbol{P}$ of the field. In four-dimensional notation: $P^{v}=(E / c, \boldsymbol{P})=\frac{1}{c} \int_{V} d^{3} x \Theta^{0 v}(x)=$ const. Another important application involves Lorentz invariance or invariance under spatial rotations. The corresponding integral conservation law 
is a constant of motion given by the antisymmetric tensor

$$
M_{\nu \lambda}=\int d^{3} x\left[\Theta_{0 \lambda} x_{v}-\Theta_{0 v} x_{\lambda}+\frac{\partial \mathcal{L}}{\partial\left(\partial^{0} \phi_{r}\right)}\left(I^{\nu \lambda}\right)_{r s} \phi_{s}(x)\right] .
$$

For spatial rotations where $v, \lambda=1,2,3$, this relation is split into two parts, i.e., $M_{n l}=L_{n l}+S_{n l}$, with $L_{n l}$ representing the orbital angular momentum and $S_{n l}$ which describes the spin angular momentum. A third application consists of internal symmetries of the system. This yields the conserved current. Being in general not symmetric, the canonical energy-momentum tensor can be symmetrized by adding a 4-divergence.

Greiner (2000) examined and obtained the solution of the free Dirac equation (that is, the Dirac equation without potentials). The derivation of the time-evolution factor $\varepsilon=\lambda E_{p}$, where $E_{p}$ is the energy, gives rise to positive and negative energy solutions. For every momentum $p$ there are two different kinds of solutions, those with $\lambda=+1\left(\varepsilon=+E_{p}\right)$ and those with $\lambda=-1\left(\varepsilon=-E_{p}\right)$. The free one-particle states can also be classified using the helicity, which, physically, is interpreted as the projection of the fermion spin onto the momentum direction, and is a constant of motion of a free Dirac particle. It can be shown that helicity is invariant under spatial rotations. If the electron wave propagates in the direction of the $z$-axis, we have $\boldsymbol{p}=(0,0, p)$ and the helicity operator is $\hat{\Lambda}_{S}=\hat{S}_{z}=\frac{\hbar}{2} \hat{\Sigma}_{z}=\frac{\hbar}{2}\left(\begin{array}{cccc}1 & 0 & 0 & 0 \\ 0 & -1 & 0 & 0 \\ 0 & 0 & 1 & 0 \\ 0 & 0 & 0 & -1\end{array}\right)$, where $\hbar=\frac{h}{2}$ is Planck constant. One then can classify completely the free Dirac waves propagating in the $z-$ direction. However, for our interest in this paper, we will restrict ourselves to the solutions for positive energies only (where $\lambda=+1$ ). Denoting them by $\Psi_{p_{z}, \lambda, S_{z}}(x, t)$, we have explicitly,

$$
\begin{aligned}
& \Psi_{p, \lambda,+1 / 2}=N\left(\begin{array}{c}
\left(\begin{array}{c}
1 \\
0
\end{array}\right) \\
\left.\frac{c \hat{\sigma}_{z} p}{m_{0} c^{2}+\lambda E_{p}}\left(\begin{array}{l}
1 \\
0
\end{array}\right)\right) \exp \left[i\left(p z-\lambda E_{p} t\right) / \hbar\right], \\
\Psi_{p, \lambda,-1 / 2}=N\left(\begin{array}{c}
0 \\
1
\end{array}\right) \\
\frac{c \hat{\sigma}_{z} p}{m_{0} c^{2}+\lambda E_{p}}\left(\begin{array}{l}
0 \\
1
\end{array}\right)
\end{array}\right) \exp \left[i\left(p z-\lambda E_{p} t\right) / \hbar\right],
\end{aligned}
$$

where $N=\left(\frac{m_{0} c^{2}+\lambda E_{p}}{2 \lambda E_{p}}\right)^{1 / 2}$ is a normalization factor determined from the condition

$$
\int \Psi_{p \lambda}^{\dagger}(x, t) \Psi_{p^{\prime} \lambda^{\prime}}(x, t) d^{3} x=\delta_{\lambda \lambda^{\prime}} \delta\left(\boldsymbol{p}-\boldsymbol{p}^{\prime}\right) .
$$

Now, by Greiner (2000) and Guidry (1991), we study the restrictions in which a single-particle interpretation of the Dirac equation and its solutions, holds.

\section{Single-Particle Interpretation of the Plane (Free) Dirac Waves}

The Lagrange density which leads to the free Dirac equation is

$$
\mathcal{L}=i \hbar \Psi^{\dagger} \frac{\partial}{\partial t} \Psi+i \hbar c \Psi^{\dagger} \nabla \cdot \hat{\alpha} \Psi-m_{0} c^{2} \Psi^{\dagger} \hat{\beta} \Psi
$$

From this result, we can calculate the energy of the states related to the free solutions (3) and (4) of the Dirac equation:

$$
E=\varepsilon= \pm E_{p}
$$

What is the interpretation of these positive and negative energy solutions, since there is no precedent in the framework of a one-particle theory? The answer to this query is found in the proposal offered by the hole theory, whereby real electrons are assumed to be described by only positive energy states with $E=+E_{p}$. All states of negative energy are occupied by electrons, one electron in each state of negative energy and given spin projection (see Figure 1). Thus, in this way, a real electron of positive energy is also prevented from falling into energetically lower and lower states by radiation emission. This prevents a radiation catastrophe, by the effective Pauli exclusion principle by which two identical fermions 
(for example, two electrons) cannot occupy the same state. The set of filled negative-energy states is called the Fermi sea, which is in excitation, due to quantum-fluctuations of the vacuum. From the free Dirac equation the gap between the filled negative-energy states and the positive-energy states is twice the fermion rest mass, i.e., $2 m_{0} c^{2}$, as illustrated in (Figure 1). Therefore, energy supplied to the vacuum either by virtual fluctuations, or by real physical processes such as incident radiation, may cause a fermion to be promoted from the negative-energy sea to an unoccupied positive-energy state, leaving a hole in the sea (Figure 1). Such a particle-hole excitation of the vacuum produces a positive-energy fermion and a hole in the sea that will behave, because of the negative sign on the energy, as fermion of opposite charge, an antifermion. This leads to the meaningful concept of the positron, as the antiparticle of the electron. Thus, the holetheory interpretation shows explicitly that the Dirac equation is generally not a single-particle equation; because of the particle-hole excitation of the vacuum, it is inherently a many-body equation.

However, the restriction to weak and slowly varying fields, for which a broad gap $\simeq 2 m_{0} c^{2}$ remains between the two opposite energy states, renders legitimate the use of Klein-Gordon and Dirac equations as single-particle equations.

We can therefore pursue the single-particle aspect further utilizing modified one-particle operators and the Ehrenfest's theorems according to which: in nonrelativistic quantum mechanics there is always a correspondence between a relation of operators and that of classical objects (measurable values). As an example, Newton's classical equation of motion corresponds to the operator equation $\frac{d \hat{p}}{d t}=\frac{1}{i \hbar}[\hat{p}, \hat{H}]=-\nabla U$, with $\hat{H}=\hat{p}^{2} / 2 m+U(x)$. Another example is given by the operator relation

$$
\frac{d \boldsymbol{x}}{d t}=\frac{1}{i \hbar}[\boldsymbol{x}, \hat{H}]=\frac{\hat{p}}{m_{0}},
$$

which corresponds to the classical relation between the velocity and linear momentum.

\subsection{One-particle Operators}

We introduce the sign operator $\hat{\Lambda}$,

$$
\hat{\Lambda}=\frac{\hat{H}_{f}}{\sqrt{\hat{H}_{f}^{2}}}=\frac{c \hat{\alpha} \cdot \hat{p}+\hat{\beta} m_{0} c^{2}}{c \sqrt{\hat{p}^{2}+m_{0}^{2} c^{2}}} \equiv \frac{c(\hat{\alpha} \cdot \hat{p})+\hat{\beta} m_{0} c^{2}}{E_{p}},
$$

which commutes with the free Dirac Hamiltonian $\hat{H}_{f}$, and is Hermitian and unitary. $\hat{\Lambda}$ has as an eigenvalue the sign $\lambda$ $(= \pm 1)$ of the time evolution factor, with $\lambda=+1$ meaning positive-energy states, and $\lambda=-1$ negative-energy states. An arbitrary state with fixed $\lambda$ can be written in the form

$$
\Psi_{\lambda}=\sum_{S_{z}} \int A_{S_{z}}(\boldsymbol{p}) \Psi_{p, \lambda, S_{z}} d^{3} \boldsymbol{p} \Rightarrow \hat{\Lambda} \Psi_{\lambda}=\sum_{S_{z}} \int A_{S_{z}}(\boldsymbol{p}) \frac{\hat{H}_{f}}{E_{p}} \Psi_{p, \lambda, S_{z}} d^{3} \boldsymbol{p}=\lambda \Psi_{\lambda}
$$

Then the projection operators $\hat{\Lambda}_{ \pm}$is introduced in terms of $\hat{\Lambda}$ as

$$
\hat{\Lambda}_{ \pm}=\frac{1}{2}(1 \pm \hat{\Lambda})
$$

The operators $\hat{\Lambda}_{ \pm}$split off the positive (or negative) parts of the states to which they are applied. Operators are identified as "even" or "odd" according as they transform positive (negative) functions into positive (negative) or negative (positve) functions, respectively. The product of two even or two odd operators is always an even operator, and the product of an even and an odd operator is always an odd operator. Since all positive functions $(\lambda=+1)$ are orthogonal with respect to all negative $(\lambda=-1)$ functions, the expectation value of an odd operator with states of fixed $\lambda$ is always zero; hence, $\left\langle\Psi_{\lambda}\left|\hat{A}_{\text {odd }}\right| \Psi_{\lambda}\right\rangle=0$. A consistent one-particle theory can only use states with specified sign (either $\lambda=+1$ or $\lambda=-1$ ), because the energy can only be defined meaningfully in that way. However, it follows that in a consistent one-particle theory all physical quantities must necessarily be defined by even operators. It can be shown for Dirac's theory, that Ehrenfest's theorems follow under this condition too, i.e., the quantum-mechanical operator equations and the corresponding classical equations become identical. That is to say the mean values comply with the classical equations, a quite significant fact. Formalizing these considerations, we split up every operator $\hat{A}$ into an even $[\hat{A}]$ and an odd $\{\hat{A}\}$ part.

\subsection{Canonical Energy-momentum Tensor of the Free Dirac Equation}

From the Dirac Lagrange density given by (6), one can calculate the canonical energy-momentum tensor:

$$
T_{v}^{\mu}=\frac{\partial \mathcal{L}}{\partial\left(\partial_{\mu} \psi\right)} \partial_{\nu} \psi+\frac{\partial \mathcal{L}}{\partial\left(\partial_{\mu} \bar{\psi}\right)} \partial_{\nu} \bar{\psi}-\delta_{\nu}^{\mu} \mathcal{L}=\bar{\psi} i \hbar c \gamma^{\mu} \partial_{\mu} \psi-\delta_{\nu}^{\mu} \bar{\psi} i \hbar c \gamma^{\sigma} \partial_{\sigma} \psi+\delta_{\nu}^{\mu} m_{0} c^{2} \bar{\psi} \psi
$$


From this, one obtains the energy density $T_{0}^{0}$,

$$
T_{0}^{0},=-\psi^{\dagger} i \hbar \hat{\alpha} \cdot \nabla c \psi+m_{0} c^{2} \bar{\psi} \psi=\psi^{\dagger} \hat{H}_{f} \psi
$$

Consequently

$$
E=\int T_{0}^{0} d^{3} x=\left\langle\psi\left|\hat{H}_{f}\right| \psi\right\rangle,
$$

that is, the energy is just the expectation value of the free Dirac Hamiltonian $\hat{H}_{f}$ in the state $\psi$. By analogy the momentum density $T_{i}^{0}$ is given by

$$
T_{i}^{0}=\bar{\psi} i \hbar c \gamma^{0} \partial_{i} \psi=\psi^{\dagger}(\hat{p})_{i} c \psi
$$

in other words, $p_{i}=(1 / c) \int T_{i}^{0} d^{3} x=\left\langle\psi\left|(\hat{p})_{i}\right| \psi\right\rangle$ is the expectation value of the momentum operator in the state $\psi$. The components

$$
T_{j}^{i}=\bar{\psi} i \hbar c\left(\gamma^{j} \partial_{j}-\delta_{j}^{i} \gamma^{\mu} \partial_{\mu}\right) \psi+\delta_{j}^{i} m_{0} c^{2} \bar{\psi} \psi
$$

which can simply be written for each solution of the equation of motion as

$$
T_{j}^{i}=\bar{\psi} i \hbar c \gamma^{i} \partial_{j} \psi=-\bar{\psi} \gamma^{i} \hat{p}_{j} c \psi=-\psi^{\dagger} \hat{\alpha}^{i} \hat{p}_{j} c \psi,
$$

are the components of the stress-strain tensor. The trace of $T_{v}^{\mu}$ is given by

$$
T \equiv T_{\mu}^{\mu}=\bar{\psi} i \hbar c\left(\gamma^{\mu} \partial_{\mu}-4 \gamma^{\sigma} \partial_{\sigma}\right) \psi+4 m_{0} c^{2} \bar{\psi} \psi=m_{0} c^{2} \bar{\psi} \psi
$$

\subsection{Energies of the Solutions to the Dirac Equation in the Canonical Formalism}

We calculate in the framework of the canonical formalism the energies of the solutions (3) and (4) of the Dirac equation. To start with, the energy can be determined [Cf. eq. (14)] as the integral

$$
E=\int_{V} T_{0}^{0} d^{3} x
$$

If we insert (13) as well as the solutions (3) into (19), we obtain

$$
E=N^{2} \int_{V}\left(1,0, \frac{p c}{m_{0} c^{2}+\lambda E_{p}}, 0\right)\left(\hat{\alpha} \cdot \hat{p} c+\hat{\beta} m_{0} c^{2}\right)\left(\begin{array}{c}
1 \\
0 \\
\frac{p c}{m_{0} c^{2}+\lambda E_{p}} \\
0
\end{array}\right) d^{3} x
$$

Since for (3) and (4) $\boldsymbol{p}=(0,0, p)$ holds, by calculation, we obtain

$$
\begin{aligned}
E & =N^{2} V\left(1,0, \frac{p c}{m_{0} c^{2}+\lambda E_{p}}, 0\right)\left[p c\left(\begin{array}{cc}
0 & \sigma_{z} \\
\sigma_{z} & 0
\end{array}\right)+m_{0} c^{2}\left(\begin{array}{cc}
\mathbf{1} & 0 \\
0 & \mathbf{- 1}
\end{array}\right)\right]\left(\begin{array}{c}
1 \\
0 \\
\frac{p c}{m_{0} c^{2}+\lambda E_{p}} \\
0
\end{array}\right) \\
& =N^{2} V\left(m_{0} c^{2}+\lambda E_{p}\right)^{-1} 2 \lambda^{2} E_{p}^{2} .
\end{aligned}
$$

Here we have used wave functions which are normalized with respect to a finite spherical volume $V$. If we furthermore insert the expression of $N$ from above: $N^{2}=V^{-1}\left(\frac{2 \lambda E_{P}}{m_{0} c^{2}+\lambda E_{p}}\right)^{-1}$, we obtain

$$
E=\lambda E_{p}= \pm \sqrt{p^{2} c^{2}+m_{0}^{2} c^{4}}
$$

for the upper energy continuum $(\lambda=+1)$ and for the lower energy continuum $(\lambda=-1)$. Hence, the free Dirac equation leads to states with positive and negative energy.

As for the Dirac Hamiltonian $\hat{H}_{f}$, the momentum operator component $\hat{p}$ is also obtained:

$$
\hat{\Lambda} \hat{p} \hat{\Lambda}=\hat{p}, \text { so that }[\hat{p}]=\hat{p} .
$$


For the even part of the $\hat{\alpha}$ operator component, we have

$$
\hat{\Lambda} \hat{\alpha}_{i} \hat{\Lambda}=\frac{c \hat{\alpha} \cdot \hat{p}+\hat{\beta} m_{0} c^{2}}{c \sqrt{\hat{p}^{2}+m_{0}^{2} c^{2}}} \hat{\alpha}_{i} \frac{c \hat{\alpha} \cdot \hat{p}+\hat{\beta} m_{0} c^{2}}{c \sqrt{\hat{p}^{2}+m_{0}^{2} c^{2}}}=-\hat{\alpha}_{i}+2 c \hat{p}_{i} \frac{\hat{\Lambda}}{c \sqrt{\hat{p}^{2}+m_{0}^{2} c^{2}}},
$$

and therefore

$$
\left[\hat{\alpha}_{i}\right]=\frac{1}{2}\left(\hat{\alpha}_{i}+\hat{\Lambda} \hat{\alpha}_{i} \hat{\Lambda}\right)=c \hat{p}_{i} \frac{\hat{\Lambda}}{c \sqrt{\hat{p}^{2}+m_{0}^{2} c^{2}}} .
$$

Similarly

$$
[\hat{\beta}]=\frac{m_{0} c^{2}}{E_{p}} \hat{\Lambda}=m_{0} c^{2} \frac{\hat{\Lambda}}{c \sqrt{\hat{p}^{2}+m_{0}^{2} c^{2}}} .
$$

Now we determine the velocity operator in the Dirac theory. Since we are concerned with an equation of Shrôdinger type, the theorems for the time derivatives of operators which are formulated in (8) are also valid here, and we obtain

$$
\begin{aligned}
\frac{d \hat{x}}{d t} & =\frac{1}{i \hbar}\left[\hat{x}, \hat{H}_{f}\right]=\frac{1}{i \hbar}\left[\hat{x}, c \hat{\alpha} \cdot \hat{p}+\hat{\beta} m_{0} c^{2}\right] \\
& =\frac{c}{i \hbar}[\hat{x}, \hat{\alpha} \cdot \hat{p}]=c \hat{\alpha} \equiv \hat{v} .
\end{aligned}
$$

Since the eigenvalues of $\hat{\alpha}$ have the values \pm 1 , we obtain here the "seeming" paradoxical result that the absolute value of the velocity of a relativistic spin-1/2 particle always equals the velocity of light. ${ }^{1}$ Of course, one may observe that the components of the velocity $d x_{i} / d t$ would not be simultaneously measurable since the $\hat{\alpha}_{i}$ do not commute with each other. Nevertheless, in the nonrelativistic limit where the Dirac equation is examined for physically reasonable results, this "phenomenon" is confirmed, appealing for further investigation.

\subsection{The Symmetrized Dirac Lagrange Density}

In order to investigate the possibility of free Dirac particles superluminal motion introduced by the above paradox, we turn to Greiner \& Reinhardt (1996), for a short review of some fundamental relations of spin-1/2 fields (which are described by the Dirac equation). Consider the Dirac wave function $\psi(x, t)$ as a classical field and derive the corresponding wave equation from a Lagrange function.

With the covariant relativistic notation, the Dirac equation for massive spin- $1 / 2$ particles reads (in relativistic units: $\hbar=$ $c=1)$

$$
\left(i \gamma^{\mu} \partial_{\mu}-m\right) \psi=0
$$

where the four Dirac matrices $\gamma^{\mu}, \mu=0, \ldots, 3$, satisfy the algebra

$$
\gamma^{\mu} \gamma^{v}+\gamma^{v} \gamma^{\mu}=2 g^{\mu \nu}
$$

The wave function $\psi$ has four components and satisfies the transformation law of a relativistic spinor. The dimension of the Dirac field is $\operatorname{dim}[\psi]=$ length ${ }^{-3 / 2}$, i.e., it has the natural dimension $d=+3 / 2$.

As seen in equation (6) the Lagrangian that leads to (26) is a bilinear function composed of the fields $\psi, \dot{\psi}, \nabla \psi$ and the hermitian conjugate fields $\psi^{\dagger}, \dot{\psi}^{\dagger}, \nabla \psi^{\dagger}$. It has to transform as a Lorentz scalar density and can contain only derivatives of first order since the Dirac equation itself is of first order. We now show that this Lagrangian leads to the Dirac equation (26). First rewrite (6) in relativistic units as

$$
\mathcal{L}=\bar{\psi}\left(i \gamma^{\mu} \partial_{\mu}-m\right) \psi=i \psi^{\dagger} \dot{\psi}+i \psi^{\dagger} \alpha \cdot \nabla \psi-m \psi^{\dagger} \beta \psi
$$

Here, in the second step the matrices $\beta=\gamma^{0}, \beta^{2}=\mathbf{1}, \alpha=\gamma^{0} \gamma$ and the adjoint spinor $\bar{\psi}=\psi^{\dagger} \gamma^{0}$ were used. We will treat the spinors $\psi$ and $\psi^{\dagger}$ as independent fields, each having four components. Differentiation of $\mathcal{L}$ with respect to these fields and to their time and space derivatives gives

$$
\frac{\partial \mathcal{L}}{\partial \dot{\psi}}=i \psi^{\dagger}, \frac{\partial \mathcal{L}}{\partial \dot{\psi}^{\dagger}}=0, \frac{\partial \mathcal{L}}{\partial(\nabla \psi)}=i \psi^{\dagger} \alpha, \frac{\partial \mathcal{L}}{\partial\left(\nabla \psi^{\dagger}\right)}=0, \text { and } \frac{\partial \mathcal{L}}{\partial \psi}=-m \psi^{\dagger} \beta, \frac{\partial \mathcal{L}}{\partial \psi^{\dagger}}=i \dot{\psi}+i \alpha \cdot \nabla \psi-m \beta \psi
$$

\footnotetext{
${ }^{1}$ This was first recognized by Gregory Breit in 1928 .
} 
Note that these equations are to be interpreted as matrix equations. For example the last but one equation reads explicitly

$$
\frac{\partial \mathcal{L}}{\partial \psi_{\rho}}=-m \sum_{\tau} \psi_{\tau}^{\dagger} \beta_{\tau \rho}
$$

For a much more compact notation, we will drop the spinor indices without causing confusion. Variation of the action with respect to $\psi^{\dagger}$ leads to the Euler-Lagrange equation

$$
\frac{\partial}{\partial t} \frac{\partial \mathcal{L}}{\partial \dot{\psi}^{\dagger}}=\frac{\partial \mathcal{L}}{\partial \psi^{\dagger}}-\nabla \frac{\partial \mathcal{L}}{\partial\left(\nabla \psi^{\dagger}\right)} \Longrightarrow i \dot{\psi}+i \alpha \cdot \nabla-m \beta \psi=0,
$$

where we have used (29). Multiplying this last equation by $\gamma^{0}=\beta$, we obtain the standard form of the Dirac equation

$$
\left(i \gamma^{\mu} \partial_{\mu}-m\right) \psi=0
$$

as desired. Variation with respect to $\psi$ using (29) leads to the differential equation

$$
i \dot{\psi}^{\dagger}=-m \psi^{\dagger}-i \nabla \psi^{\dagger} \alpha .
$$

This can be identified as the hermitian conjugate of (31)

$$
\bar{\psi}\left(i \gamma^{\mu} \overleftarrow{\partial_{\mu}}+m\right)=0
$$

where the arrow indicates that the partial derivative acts on the function to the left.

To proceed further we need the canonically conjugate fields $\pi_{\psi}$ and $\pi_{\psi^{*}}$. As in the case of the Shrödinger field these turn out to dependent quantities. We find

$$
\pi_{\psi}=\frac{\partial \mathcal{L}}{\partial \dot{\psi}}=i \psi^{\dagger}, \quad \pi_{\psi^{\dagger}}=\frac{\partial \mathcal{L}}{\partial \dot{\psi}^{\dagger}}=0 .
$$

Thus there are two independent degrees of freedom of the Dirac field, which are given by $\psi$ and $\psi^{\dagger}$.

As usual the Hamilton density is obtained through the Legendre transformation

$$
\begin{aligned}
\mathcal{H} & =\pi_{\psi} \dot{\psi}+\pi_{\psi^{\dagger}} \dot{\psi}^{\dagger}-\mathcal{L}=i \psi^{\dagger} \dot{\psi}-i \psi^{\dagger} \dot{\psi}-i \psi^{\dagger} \alpha \cdot \nabla \psi+m \psi^{\dagger} \beta \psi \\
& =\psi^{\dagger}(-i \alpha \cdot \nabla+\beta m) \psi .
\end{aligned}
$$

The Hamiltonian is thus given by the expectation value of Dirac's differential operator $H_{D}=\alpha \cdot \boldsymbol{p}+\beta m$ :

$$
H=\int d^{3} x \psi^{\dagger}(x, t)(-i \alpha \cdot \nabla+\beta m) \psi(x, t) .
$$

Finally let us derive the conserved quantities that follow from Noether's theorem. The canonical energy-momentum tensor $\Theta_{\mu \nu}$ reads

$$
\Theta_{\mu \nu}=\frac{\partial \mathcal{L}}{\partial\left(\partial^{\mu} \psi\right)} \partial_{\nu} \psi+\frac{\partial \mathcal{L}}{\partial\left(\partial^{\mu} \psi^{\dagger}\right)} \partial_{\nu} \psi^{\dagger}-g_{\mu \nu} \mathcal{L}=\bar{\psi} i \gamma_{\mu} \partial_{\nu} \psi-g_{\mu \nu} \bar{\psi}\left(i \gamma^{\sigma} \partial_{\sigma}-m\right) \psi,
$$

which leads to the conserved four-momentum vector

$$
P_{v}=\int d^{3} x \Theta_{0 v}=\int d^{3} x\left[\bar{\psi} i \gamma_{0} \partial_{\nu} \psi-g_{0 v} \bar{\psi}\left(i \gamma^{\sigma} \partial_{\sigma}-m\right) \psi\right] .
$$

The time component of these vector is the energy

$$
P_{0}=\int d^{3} x \bar{\psi}\left(i \gamma_{0} \partial_{0}-i \gamma_{0} \partial_{0}-i \gamma \cdot \nabla+m\right) \psi=\int d^{3} x \psi^{\dagger}(-i \alpha \cdot \nabla+\beta m) \psi
$$

As expected this is just the Hamiltonian $H$ of (37). The spatial momentum vector reads

$$
\boldsymbol{P}=-i \int d^{3} x \psi^{\dagger} \nabla \psi
$$

The transformation law of Dirac spinors under infinitesimal Lorentz transformations is given by

$$
\psi^{\prime}\left(x^{\prime}\right)=\psi(x)-\frac{i}{4} \delta \omega_{\mu \nu} \sigma^{\mu v} \psi(x)
$$


where $\sigma^{\mu \nu}=\frac{i}{2}\left[\gamma^{\mu}, \gamma^{\nu}\right]$. This leads to the infinitesimal generators

$$
\left(I^{\mu v}\right)_{\alpha \beta}=-\frac{i}{2}\left(\sigma^{\mu v}\right)_{\alpha \beta},
$$

where $\mu, v=0, \ldots, 3$ are Lorentz indices and $\alpha, \beta=1, \ldots, 4$ are Dirac indices. The generalized angular momentum tensor of the Dirac field has the form

$$
\begin{gathered}
M_{v \lambda}=L_{v \lambda}+S_{v \lambda}, \\
L_{v \lambda}=\int d^{3} x\left(\Theta_{0 \lambda} x_{v}-\Theta_{0 v} x_{\lambda}\right), \\
S_{v \lambda,}=\int d^{3} x \frac{\partial \mathcal{L}}{\partial\left(\partial^{0} \psi\right)} I_{v \lambda} \psi=\frac{1}{2} \int d^{3} x \psi^{\dagger} \sigma_{v \lambda} \psi .
\end{gathered}
$$

The three-dimensional vectors of orbital and spin angular momentum are

$$
\begin{gathered}
\boldsymbol{L}=-i \int d^{3} x \psi^{\dagger} \boldsymbol{x} \times \boldsymbol{\nabla} \psi, \\
\boldsymbol{S}=\frac{1}{2} \int d^{3} x \psi^{\dagger} \boldsymbol{\Sigma} \psi .
\end{gathered}
$$

In the standard representation of the Dirac matrices, $\boldsymbol{\Sigma}$ is the "double" Pauli matrix

$$
\Sigma=\left(\begin{array}{cc}
\sigma & 0 \\
\mathbf{0} & \sigma
\end{array}\right)
$$

The Lagrange density (28) is invariant under global phase transformations $\psi \rightarrow \psi e^{i \chi}$ and $\psi^{\dagger} \rightarrow \psi^{\dagger} e^{-i \chi}$. This implies the existence of conserved current-density vector $j_{\mu}(x, t)$ :

$$
j_{\mu}=-i e\left(\frac{\partial \mathcal{L}}{\partial\left(\partial^{\mu} \psi\right)} \psi-\frac{\partial \mathcal{L}}{\partial\left(\partial^{\mu} \psi^{\dagger}\right)} \psi^{\dagger}\right)=e \bar{\psi} \gamma_{\mu} \psi
$$

We have included the electrical elementary charge $e$ as a factor in the definition of $j_{\mu}$ since expression (50) is just the electrical current density of the Dirac field. The resulting conserved quantity is the total charge

$$
Q=\int d^{3} x j_{0}(x, t)=e \int d^{3} x \psi^{\dagger} \psi
$$

The constancy of $Q$ is seen to coincide with the conservation of the norm of the Dirac field.

Next we show that $\mathcal{L}$ is not a real number, since

$$
\begin{aligned}
\mathcal{L}^{\star} & \equiv \mathcal{L}^{\dagger}=\left[\bar{\psi}\left(i \gamma^{\mu} \overrightarrow{\partial_{\mu}}-m\right) \psi\right]^{\dagger}=\psi^{\dagger}\left(-i\left(\gamma^{\mu}\right)^{\dagger} \overleftarrow{\partial_{\mu}}-m\right)\left(\gamma^{0}\right)^{\dagger} \psi \\
& =\psi^{\dagger}\left(-i \gamma^{0} \gamma^{\mu} \gamma^{0} \overleftarrow{\partial_{\mu}}-m\right) \gamma^{0} \psi=\bar{\psi}\left(-i \gamma^{\mu} \overleftarrow{\partial_{\mu}}-m\right) \psi,
\end{aligned}
$$

where the properties of the $\gamma$ matrices $\left(\gamma^{0}\right)^{\dagger}=\gamma^{0}$ and $\gamma^{0}\left(\gamma^{\mu}\right)^{\dagger} \gamma^{0}=\gamma^{\mu}$ have been used. The expression (52) in general will not agree with (28), $\mathcal{L}^{\star} \neq \mathcal{L}$. This deficiency, however, can be cured by defining the real Lagrange density as

$$
\begin{aligned}
\mathcal{L}^{\prime}: & =\frac{1}{2}\left(\mathcal{L}+\mathcal{L}^{\dagger}\right)=\frac{1}{2} \bar{\psi}\left(i \gamma^{\mu} \overrightarrow{\partial_{\mu}}-m\right) \psi+\frac{1}{2} \bar{\psi}\left(-i \gamma^{\mu} \overleftarrow{\partial_{\mu}}-m\right) \psi \\
& =\frac{1}{2} \bar{\psi} i \gamma^{\mu} \overleftrightarrow{\partial_{\mu}} \psi-m \bar{\psi} \psi
\end{aligned}
$$

This symmetrized Lagrange density essentially leads to the same equations of motion and to the same conserved quantities as the simpler relation (28). We show this in Theorem 1, below.

Theorem 1 The symmetrized Dirac Lagrange density (53) leads to the same equations of motion and the same conservation laws as the unsymmetrized expression (28). 
Proof. This can be seen even without performing the explicit calculation since $\mathcal{L}^{\prime}$ and $\mathcal{L}$ are identical up to a fourdivergence:

$$
\mathcal{L}^{\prime}=\mathcal{L}+\delta \mathcal{L}=\mathcal{L}+\frac{1}{2}(-i) \partial^{\mu}\left(\bar{\psi} \gamma_{\mu} \psi\right)
$$

This additional term $\delta \mathcal{L}$ only leads to a surface term in the action integral and thus does not show up in the Euler-Lagrange equation.

To compute the conserved quantities the canonically conjugate fields are needed:

$$
\pi_{\psi}=\frac{\partial \mathcal{L}^{\prime}}{\partial \dot{\psi}}=\frac{i}{2} \psi^{\dagger} \equiv \pi \quad \text { and } \quad \pi_{\psi^{\dagger}}=\frac{\partial \mathcal{L}^{\prime}}{\partial \dot{\psi}^{\dagger}}=-\frac{i}{2} \psi \equiv \pi^{\dagger} .
$$

The energy-momentum tensor then becomes

$$
\Theta_{0 v}^{\prime}=\pi \partial_{\nu} \psi+\pi^{\dagger} \partial_{\nu} \psi^{\dagger}-g_{0 v} \mathcal{L}^{\prime}=\frac{i}{2} \psi^{\dagger} \overleftrightarrow{\partial_{\nu}} \psi-g_{0 v}\left(\frac{i}{2} \bar{\psi} \gamma^{\mu} \overleftrightarrow{\partial_{\mu}} \psi-m \bar{\psi} \psi\right)
$$

The difference between $\Theta_{0 v}^{\prime}$ and the original result $\Theta_{0 v}$ given in (38) is

$$
\delta \Theta_{0 v}=\Theta_{0 v}^{\prime}-\Theta_{0 v}=-\frac{i}{2} \partial_{v}\left(\psi^{\dagger} \psi\right)+\frac{i}{2} g_{0 v} \partial_{\mu}\left(\bar{\psi} \gamma^{\mu} \psi\right) .
$$

This implies that there is no difference in the field energy:

$$
\begin{aligned}
\delta P_{0} & =\int d^{3} x \delta \Theta_{00}=\int d^{3} x\left[-\frac{i}{2} \partial_{0}\left(\psi^{\dagger} \psi\right)+\frac{i}{2} \partial_{0}\left(\bar{\psi} \gamma^{0} \psi\right)+\frac{i}{2} \nabla \cdot(\bar{\psi} \gamma \psi)\right] \\
& =\frac{i}{2} \int d^{3} x \boldsymbol{\nabla} \cdot\left(\psi^{\dagger} \alpha \psi\right)=0 .
\end{aligned}
$$

In the same way the space components $(n=1,2,3)$ of the momentum differ only by a vanishing surface term

$$
\delta P_{n}=-\frac{i}{2} \int d^{3} x \partial_{n}\left(\psi^{\dagger} \psi\right)=0 .
$$

The generalized angular momentum tensor becomes

$$
M_{v \lambda}^{\prime}=L_{v \lambda}^{\prime}+S_{v \lambda}^{\prime}
$$

where

$$
L_{\nu \lambda}^{\prime}=\int d^{3} x\left(\Theta_{0 \lambda}^{\prime} x_{v}-\Theta_{0 v}^{\prime} x_{\lambda}\right)=L_{\nu \lambda}+\int d^{3} x\left(\delta \Theta_{0 \lambda} x_{v}-\delta \Theta_{0 v} x_{\lambda}\right)=L_{v \lambda}+\delta L_{\nu \lambda} .
$$

The difference in the spatial orbital angular momentum tensor $(v=n, \lambda=l)$ again vanishes:

$$
\begin{aligned}
\delta L_{n l} & =\int d^{3} x\left[-\frac{i}{2} \partial_{l}\left(\psi^{\dagger} \psi\right) x_{n}+\frac{i}{2} \partial_{n}\left(\psi^{\dagger} \psi\right) x_{l}\right] \\
& =\int d^{3} x\left[-\frac{i}{2} \partial_{l}\left(\psi^{\dagger} \psi x_{n}\right)-\frac{i}{2} \psi^{\dagger} \psi g_{l n}+\frac{i}{2} \partial_{n}\left(\psi^{\dagger} \psi x_{l}\right)+\frac{i}{2} \psi^{\dagger} \psi g_{n l}\right]=0
\end{aligned}
$$

The mixed spacetime components, however, do not vanish:

$$
\begin{aligned}
\delta L_{0 l} & =\int d^{3} x\left(\delta \Theta_{0 l} x_{0}-\delta \Theta_{00} x_{l}\right)=-\frac{i}{2} \int d^{3} x\left[\partial_{l}\left(\psi^{\dagger} \psi\right) x_{0}+\partial_{k}\left(\bar{\psi} \gamma^{k} \psi\right) x_{l}\right] \\
& =-\frac{i}{2} \int d^{3} x\left[\partial_{k}\left(\bar{\psi} \gamma^{k} \psi x_{l}\right)-\bar{\psi} \gamma^{k} \psi g_{k l}\right]=\frac{i}{2} \int d^{3} x \bar{\psi} \gamma_{l} \psi \neq 0 .
\end{aligned}
$$

To compute the spin contribution $S_{v \lambda}^{\prime}$ in (44), the hermitian conjugate of (42) is needed:

$$
\psi^{\prime}\left(x^{\prime}\right)=\psi^{\dagger}(x)+\frac{1}{2} \Delta \omega^{\mu v} \psi^{\dagger}(x) I_{\mu \nu}^{\dagger},
$$


which according to (43) leads to

$$
S_{v \lambda}^{\prime}=\int d^{3} x\left(\pi I_{\nu \lambda} \psi+\psi^{\dagger} I_{v \lambda}^{\dagger} \pi^{\dagger}\right)=\frac{1}{4} \int d^{3} x\left(\psi^{\dagger} \sigma_{\nu \lambda} \psi+\psi^{\dagger} \sigma_{\nu \lambda}^{\dagger} \pi\right) .
$$

The space components satisfy

$$
\sigma_{n l}^{\prime}=\left[\frac{i}{2}\left(\gamma_{n} \gamma_{l}-\gamma_{l} \gamma_{n}\right)\right]^{\dagger}=-\frac{i}{2}\left(\gamma \dagger l \gamma_{n}^{\dagger}-\gamma_{n}^{\dagger} \gamma_{l}^{\dagger}\right)=\sigma_{n l}
$$

which implies

$$
S_{n l}^{\prime}=\frac{1}{2} \int d^{3} x \psi^{\dagger} \sigma_{n l} \psi=S_{n l} .
$$

The mixed components, on the other hand, vanish identically since

$$
\delta S_{0 l}=-\frac{1}{2} \int d^{3} x \psi^{\dagger} \sigma_{0 l} \psi=-\frac{1}{2} \int d^{3} x \psi^{\dagger} i \gamma_{0} \gamma_{l} \psi
$$

and

$$
\sigma_{0 l}^{\dagger}=\left[\frac{i}{2}\left(\gamma_{0} \gamma_{l}-\gamma_{l} \gamma_{0}\right)\right]^{\dagger}=-\sigma_{0 l}
$$

thus

$$
S_{0 l}^{\prime}=S_{0 l}+\delta S_{0 l}=0
$$

Therefore the differences in the orbital and spin angular momentum are found to cancel each other:

$$
\delta M_{0 l}=\delta L_{0 l}+\delta S_{0 l}=\frac{i}{2} \int d^{3} x \bar{\psi} \gamma_{l} \psi-\frac{1}{2} \int d^{3} x \psi^{\dagger} i \gamma_{0} \gamma_{l} \psi=0
$$

At this point, we reach the limit of our review of results sufficient for the purpose of this work. In the following, for our investigation, we propose, with no essential lost of generality, to restrict ourselves to realistic one-particle systems with positive energy, and we use the free Dirac waves propagating in the $z$-direction.
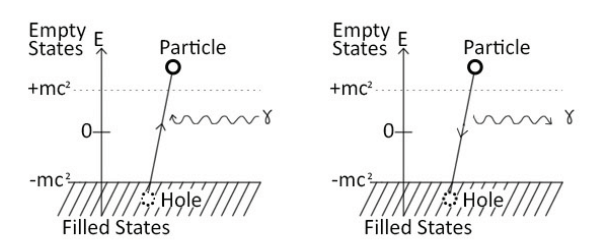

Figure 1. Gap between the filled negative-energy states and the positive-energy states.

\section{Superluminal Motion of Free Spin-1/2 Particles in Spacetime}

We utilize the symmetrized Dirac Lagrange density. Our interest here concerns two results of Theorem 1; they are eq.(63) of variations in mixed space-time components of orbital angular momentum, and eq.(68) of spin angular momentum. Consider waves propagating in the $z$-direction so that the index $l=3$; we rewrite, using functional notation (Gelfand \& Fomin (1991)), these equations as

$$
\begin{gathered}
\delta L_{03}\left[\psi(x), \psi^{\dagger}(x)\right]=S_{03}\left[\psi(x), \psi^{\dagger}(x)\right]=\frac{1}{2} \int d^{3} x \psi^{\dagger}(x) \sigma_{03} \psi(x) \\
\delta S_{03}\left[\psi(x), \psi^{\dagger}(x)\right]=-S_{03}\left[\psi(x), \psi^{\dagger}(x)\right]=-\frac{1}{2} \int d^{3} x \psi^{\dagger}(x) \sigma_{03} \psi(x)
\end{gathered}
$$






Figure 2. Causality violation by variation in spin angular momentum in the light-cone.

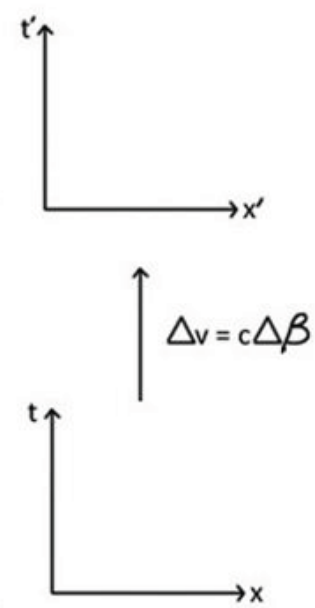

Figure 3. Two reference systems: Lorentz boost in the $z$-direction.

Inserting the solutions of the Dirac equation (3) into (71), computing and integrating, yield

$$
\begin{aligned}
& \delta L_{03}(x)=\frac{1}{2} \int d^{3} x N\left(\begin{array}{cccc}
1 & 0 & \frac{c \hat{\sigma}_{z} p}{m_{0} c^{2}+E_{p}} & 0
\end{array}\right) \exp \left[-i\left(p z-E_{p} t\right) / \hbar\right] \\
& \times \sigma_{03} N\left(\begin{array}{c}
\left(\begin{array}{c}
1 \\
0
\end{array}\right) \\
\frac{c \hat{\sigma}_{z} p}{m_{0} c^{2}+E_{p}}\left(\begin{array}{l}
1 \\
0
\end{array}\right)
\end{array}\right) \exp \left[i\left(p z-E_{p} t\right) / \hbar\right] \\
& =\frac{1}{2} \sigma_{03} x_{\mu}=\frac{1}{2} \sigma_{03}\left(\begin{array}{c}
t \\
x \\
y \\
z
\end{array}\right) \text {. }
\end{aligned}
$$

Thus, $\delta L_{03}(x)$ is a linear dependence on the 4-vector $x_{\mu}=\left(\begin{array}{c}t \\ x \\ y \\ z\end{array}\right)$ alone, along the $z-$ axis. Similarly, (72) simplifies to

$$
\delta S_{03}(x)=-\frac{1}{2} \sigma_{03} x_{\mu}=\frac{1}{2} \sigma_{03}\left(-x_{\mu}\right)=\frac{1}{2} \sigma_{03}\left(\begin{array}{c}
-t \\
-x \\
-y \\
-z
\end{array}\right)
$$






Figure 4. Electrons with positive and negative helicity: the double arrow denotes spin-up and spin-down and symbolizes the two possible rotations of the electron.

So, variation in spin angular momentum in (75) leads to negative temporal component $(t \rightarrow-t)$, depicting backward in time motion (Figure 2): Clearly, physically interpreted, this is causality violation. Shore (2003) and Modanese (2013) discuss the velocity requirements for the possibility of such an occurrence. There exist suitable reference systems in relative motion where the temporal order of events appears to be reversed, i.e., the delay $\Delta t^{\prime}$ becomes negative; this is mathematically due to the singularity of the Lorentz transformations for $\beta \rightarrow 1^{-}$. But then, a legitimate question arises as to how fast must such a relative motion be, for the moving observer to see the temporal order of the two events reversed. It has been shown that if the two events appear to be connected by a superluminal signal with velocity $V$ (i.e., $\Delta x / \Delta t=V>c$ in the rest reference system), then the causality violation is observed in reference systems moving with relative velocity $v$ larger than $c^{2} / V$, Modanese (2013). As a result, it is legitimate to regard the phenomenon described by (74) as a superluminal propagation. In contradistinction, variation in orbital angular momentum does not violate causality and so accords with special relativistic principles of subluminal motion.

We will now compute and identify the transformations which underpin these events.

\subsection{Evaluation of the Dirac Spinor $\sigma_{03}$ in Eqs. (74)-(75)}

From the transformation law of Dirac spinors under infinitesimal Lorentz transformations given through (42)-(43) above, we have in particular

$$
\begin{aligned}
\sigma^{0 k} & =\frac{i}{2}\left(\gamma^{0} \gamma^{k}-\gamma^{k} \gamma^{0}\right)=i \gamma^{0} \gamma^{k}=i \alpha^{k}=i\left(\begin{array}{cc}
0 & \sigma_{k} \\
\sigma_{k} & 0
\end{array}\right), \\
\sigma^{i j} & =\epsilon^{i j k} \Sigma_{k}=\epsilon^{i j k}\left(\begin{array}{cc}
\sigma_{k} & 0 \\
0 & \sigma_{k}
\end{array}\right)
\end{aligned}
$$

where we employ an obvious shorthand notation where each entry of the matrices is itself a $2 \times 2$ matrix, $\epsilon^{i j k}$ is the antisymmetric third-rank tensor and $\sigma_{k}$ are Pauli spin matrices (with $k=1,2,3$ ). In (76) when $k=3$, we have $\sigma^{03}=$ $i \alpha^{3}=i\left(\begin{array}{cc}0 & \sigma_{3} \\ \sigma_{3} & 0\end{array}\right)$ where $\sigma_{3}=\left(\begin{array}{cc}1 & 0 \\ 0 & -1\end{array}\right)$ represents spin around the $z-$ axis, so that

$$
\sigma^{03}=i\left(\begin{array}{cccc}
0 & 0 & 1 & 0 \\
0 & 0 & 0 & -1 \\
1 & 0 & 0 & 0 \\
0 & -1 & 0 & 0
\end{array}\right) .
$$

Next, if a contravariant 4-vector has components $A^{\mu}=\left(A^{0}, A^{1}, A^{2}, A^{3}\right)$, its covariant partner has components $A_{0}=A^{0}$, $A_{1}=-A^{1}, A_{2}=-A^{2}$, and $A_{3}=-A^{3}$. That is, if $A^{\mu}=\left(A^{0}, \mathbf{A}\right)$ then the corresponding covariant vector is $A_{\mu}=\left(A^{0},-\mathbf{A}\right)$. The 3-vector $\mathbf{A}$ (in boldface notation) has components $\left(A^{1}, A^{2}, A^{3}\right)$. It follows, symbolically, concerning the 3-vector $\alpha^{k}$ in (76), that $\alpha^{k}=\left\{\alpha^{1}, \alpha^{2}, \alpha^{3}\right\}=\left\{-\alpha_{1},-\alpha_{2},-\alpha_{3}\right\}$, Greiner (2000). Now, from the contravariant equation $\sigma^{03}=i \alpha^{3}$ in (76), we have the corresponding covariant equation $\sigma_{03}=i \alpha_{3}=-i \alpha^{3}$, that is

$$
\sigma_{03}=i\left(-\alpha^{3}\right)=i\left(\begin{array}{cccc}
0 & 0 & -1 & 0 \\
0 & 0 & 0 & 1 \\
-1 & 0 & 0 & 0 \\
0 & 1 & 0 & 0
\end{array}\right) .
$$

The polar form of the complex number $(x, y)$ or $x+i y$ in the complex plane is $w=x+i y=r(\cos \theta+i \sin \theta)$ with $x=r \cos \theta$ and $y=r \sin \theta$, where $r=\sqrt{x^{2}+y^{2}}=|x+i y|$ is called the modulus of $w$ and $\theta$ the amplitude or argument of 
$w$. Specifically, the complex number $i=1 \cdot[\cos (\pi / 2)+i \sin (\pi / 2)]$ in polar form or, $i=e^{i \pi / 2}$, using Euler's formula, is unimodular and has amplitude or argument $\theta=\pi / 2$. In this form, when it is applied (in multiplication) to any plane vector $(x, y)$, this represents a unimodular rotation transformation of angle $\theta=\pi / 2$ about the origin $\mathrm{O}$ of the $x y-$ coordinate system. Obviously, its application to a 3-vector $(x, y, z)$ in 3-space corresponds to a unimodular 3-dimensional rotation transformation of angle $\theta=\pi / 2$ about the $z$-axis; so that the matrix of the transformation is unimodular and given by $\left(\begin{array}{ccc}\cos (\pi / 2) & \sin (\pi / 2) & 0 \\ -\sin (\pi / 2) & \cos (\pi / 2) & 0 \\ 0 & 0 & 1\end{array}\right)=\left(\begin{array}{ccc}0 & 1 & 0 \\ -1 & 0 & 0 \\ 0 & 0 & 1\end{array}\right)$, which is an element of the group $S O(3)$ of $3 \times 3$ unimodular orthogonal matrices of determinant 1 . Notice that $S O(3)$ is a subgroup of $S O(1,3)$ that denotes the group of all unimodular $4 \times 4$ rotation matrices of determinant unity, also called the Lorentz group. Hence, in an extension to 4-dimensional space-time (1-time, 3-space), multiplication by the complex number $i$ corresponds to multiplying by the $4 \times 4$ matrix $\left(\begin{array}{cccc}1 & 0 & 0 & 0 \\ 0 & \cos (\pi / 2) & \sin (\pi / 2) & 0 \\ 0 & -\sin (\pi / 2) & \cos (\pi / 2) & 0 \\ 0 & 0 & 0 & 1\end{array}\right)=\left(\begin{array}{cccc}1 & 0 & 0 & 0 \\ 0 & 0 & 1 & 0 \\ 0 & -1 & 0 & 0 \\ 0 & 0 & 0 & 1\end{array}\right)$. Substituting this into $(79)$, we obtain the Dirac spinor in the z-direction:

$$
\sigma_{03}=\left(\begin{array}{cccc}
1 & 0 & 0 & 0 \\
0 & 0 & 1 & 0 \\
0 & -1 & 0 & 0 \\
0 & 0 & 0 & 1
\end{array}\right)\left(\begin{array}{cccc}
0 & 0 & -1 & 0 \\
0 & 0 & 0 & 1 \\
-1 & 0 & 0 & 0 \\
0 & 1 & 0 & 0
\end{array}\right)=\left(\begin{array}{cccc}
0 & 0 & -1 & 0 \\
-1 & 0 & 0 & 0 \\
0 & 0 & 0 & -1 \\
0 & 1 & 0 & 0
\end{array}\right)
$$

We can now compute the matrix of the variation $\delta S_{03}$. We obtain

$$
M_{\delta S_{03}}=-\frac{1}{2} \sigma_{03}=\left(\begin{array}{cccc}
0 & 0 & \frac{1}{2} & 0 \\
\frac{1}{2} & 0 & 0 & 0 \\
0 & 0 & 0 & \frac{1}{2} \\
0 & -\frac{1}{2} & 0 & 0
\end{array}\right)
$$

Similarly the matrix of the variation $\delta L_{03}$ is

$$
M_{\delta L_{03}}=\frac{1}{2} \sigma_{03}=\left(\begin{array}{cccc}
0 & 0 & -\frac{1}{2} & 0 \\
-\frac{1}{2} & 0 & 0 & 0 \\
0 & 0 & 0 & -\frac{1}{2} \\
0 & \frac{1}{2} & 0 & 0
\end{array}\right)
$$

\subsection{Determination of the 'Expectation of Trajectory' of the Free Spin-1/2 Field}

The propagation of the fermion field is 'expected' to be a helical motion and helicity is invariant under spatial rotations, Greiner (2000); Guidry (1991); Ohanian (1984) (see Figure 4). Based on Ehrenfest's theorems, the expectation of position or, precisely, the expectation of trajectory of a spin-1/2 particle corresponds to the classical 3-space circular (right-handed) helix parametrized by the equations:

$$
x_{1}=a \cos t, \quad x_{2}=a \sin t, \quad x_{3}=b t, \quad a, b \neq 0, \quad-\infty<t<\infty,
$$

which lead to the parametric derivative equations:

$$
d x_{1} / d t=-a \sin t, \quad d x_{2} / d t=a \cos t, \quad d x_{3} / d t=b .
$$

Observe that the constant $b$ represents the linear velocity component in the $z$-direction.

\subsection{Calculation of the Expectation Value of the Overall Linear Velocity Component of the Field}

Let $V_{S}$ and $v_{L}$ be the expectation values of the linear velocity components under the transformations $M_{\delta S_{03}}$ and $M_{\delta L_{03}}$, respectively. We must understand that every transformation from one reference system to another (in the light cone) is a velocity transformation or Lorentz boost (see Figure 3), from which the constant transformation matrices $M_{\delta S_{03}}$ and $M_{\delta S_{03}}$ originate. The matrices of (81) and (82) must be regarded as numerical solutions of some generalized velocity transformations which remained to be determined. Since the variations $\delta S_{03}(x)$ and $\delta L_{03}(x)$ are linear, they carry a vector $\mathbf{x}_{\mu}$ into its scalar multiples $M_{\delta S_{03}} \mathbf{x}_{\mu}$ and $M_{\delta L_{03}} \mathbf{x}_{\mu}$ under their respective matrices $M_{\delta S_{03}}$ and $M_{\delta L_{03}}$, both of which have all their entries equal in the absolute value of $1 / 2$. Furthermore, to get the clear picture of the transformation of matrix $M_{\delta S_{03}}$, observe that $M_{\delta S_{03}}$ maps the 4-vector $x_{\mu}=\left(\begin{array}{c}t \\ x \\ y \\ z\end{array}\right)$ unto its image $x_{\mu}^{\prime}=\left(\begin{array}{c}t^{\prime} \\ x^{\prime} \\ y^{\prime} \\ z^{\prime}\end{array}\right)=\left(\begin{array}{c}\frac{1}{2} y \\ \frac{1}{2} t \\ \frac{1}{2} z \\ -\frac{1}{2} x\end{array}\right)$, i.e., $t \mapsto t^{\prime}=\frac{1}{2} y, x \mapsto x^{\prime}=\frac{1}{2} t$, 
$y \mapsto y^{\prime}=\frac{1}{2} z$, and $z \mapsto z^{\prime}=-\frac{1}{2} x$. So, each coordinate of the image vector is found on a different coordinate axis, as if the coordinate axes have been altered or somehow 'interchanged' under the effect of $M_{\delta S_{03}}$. This scenario describes a quadratic (or a rotation) transformation of the coordinate axes in four dimensions. A prediction can be made of this situation from (80)-(81) where the matrix $M_{\delta S_{03}}$ has been obtained as a rotation matrix since it is the product of the Dirac Spinor $\sigma_{03}$ which is a spin (i.e., rotation) matrix and the factor $1 / 2$ which emanates from the giro factor of intrinsic spin. Bearing in mind our previous remarks, we deduce that the entries of this matrix, which are all equal in the absolute value of $1 / 2$, are expressible in a unique sine or cosine function of argument $V_{S} / c$ (representing 'boost'), where $c$ is the speed of light. This is also valid for the transformation $M_{\delta L_{03}}$. By inspection and based on our previous discussions, due to the superluminal propagation under spin, we will assign the cosine function to $M_{\delta S_{03}}$ and the sine function to the subluminal variation $\delta L_{03}$.

(1) Explicitly, for variation under spin angular momentum, we have

$$
\begin{aligned}
M_{\delta S_{03}}\left(V_{S}\right) & =\alpha_{\mu}^{v}\left(V_{S}\right)=\left(\begin{array}{cccc}
0 & 0 & \cos \left(V_{S} / c\right) & 0 \\
\cos \left(V_{S} / c\right) & 0 & 0 & 0 \\
0 & 0 & 0 & \cos \left(V_{S} / c\right) \\
0 & -\cos \left(V_{S} / c\right) & 0 & 0
\end{array}\right) \\
& =\left(\begin{array}{cccc}
0 & 0 & \frac{1}{2} & 0 \\
\frac{1}{2} & 0 & 0 & 0 \\
0 & 0 & 0 & \frac{1}{2} \\
0 & -\frac{1}{2} & 0 & 0
\end{array}\right)
\end{aligned}
$$

that is

$$
\begin{gathered}
\cos \left(V_{S} / c\right)=\frac{1}{2}, \\
\Longrightarrow V_{S}=\left(\frac{\pi}{3}+2 k \pi\right) \times c
\end{gathered}
$$

where $k=0,1,2, \ldots$ Thus, the expectation value of the relative linear velocity component under spin angular momentum is superluminal, and its minimal value is

$$
V_{S_{\min }}=(\pi / 3) c=(1.047 \ldots .) \times c,
$$

which also exceeds the speed of light.

(2) Similarly, for variation under orbital angular momentum, we have

$$
M_{\delta L_{03}}\left(v_{L}\right)=\left(\begin{array}{cccc}
0 & 0 & -\sin \left(v_{L} / c\right) & 0 \\
-\sin \left(v_{L} / c\right) & 0 & 0 & 0 \\
0 & 0 & 0 & -\sin \left(v_{L} / c\right) \\
0 & \sin \left(v_{L} / c\right) & 0 & 0
\end{array}\right)=\left(\begin{array}{cccc}
0 & 0 & -\frac{1}{2} & 0 \\
-\frac{1}{2} & 0 & 0 & 0 \\
0 & 0 & 0 & -\frac{1}{2} \\
0 & \frac{1}{2} & 0 & 0
\end{array}\right),
$$

that is

$$
\begin{gathered}
\sin \left(v_{L} / c\right)=\frac{1}{2} \\
\Longrightarrow v_{L}=\left(\frac{\pi}{6}\right) \times c \simeq(0.524) \times c .
\end{gathered}
$$

Notice here that we must ignore the additive $\pm 2 k \pi$ term in order to keep $v_{L}$ subluminal.

(3) Furthermore, let $V$ denote the expectation value of the overall relative linear velocity component of the field. It should also be understood that the transformation of the generalized angular momentum in mixed space-time components (let us denote it by $\mathcal{F}$ ) is the composition of the two transformations spin $M_{\delta S_{03}}$ and orbital $M_{\delta L_{03}}$. Based on our previous discussions, we have

$$
\begin{aligned}
& \mathcal{F}(V)=M_{\delta L_{03}} \circ M_{\delta S_{03}}=M_{\delta L_{03}} M_{\delta S_{03}} \\
& =\left(\begin{array}{cccc}
0 & 0 & -\frac{1}{2} & 0 \\
-\frac{1}{2} & 0 & 0 & 0 \\
0 & 0 & 0 & -\frac{1}{2} \\
0 & \frac{1}{2} & 0 & 0
\end{array}\right)\left(\begin{array}{cccc}
0 & 0 & \frac{1}{2} & 0 \\
\frac{1}{2} & 0 & 0 & 0 \\
0 & 0 & 0 & \frac{1}{2} \\
0 & -\frac{1}{2} & 0 & 0
\end{array}\right)=\left(\begin{array}{cccc}
0 & 0 & 0 & -\frac{1}{4} \\
0 & 0 & -\frac{1}{4} & 0 \\
0 & \frac{1}{4} & 0 & 0 \\
\frac{1}{4} & 0 & 0 & 0
\end{array}\right) \\
& =\left(\begin{array}{cccc}
0 & 0 & 0 & -\cos (V / c) \\
0 & 0 & -\cos (V / c) & 0 \\
0 & \cos (V / c) & 0 & 0 \\
\cos (V / c) & 0 & 0 & 0
\end{array}\right) \text {, }
\end{aligned}
$$


that is,

$$
\begin{gathered}
\cos (V / c)=\frac{1}{4} \\
\Longrightarrow V \simeq\left(\frac{21 \pi}{50}+2 k \pi\right) \times c, \quad(k=0,1,2, \ldots) .
\end{gathered}
$$

Hence, the expectation value of the overall relative linear velocity component of the spin-1/2 field is superluminal and, its minimal value is

$$
V_{\min }=(1.318 \ldots) \times c,
$$

this also exceeds light speed, as expected, completing our investigation.

Observe that $V$ takes discrete values (one at a time with respect to $k=0,1,2 \ldots$ ). Each value of $V$ may be thought of as corresponding to the level at which the electron is spinning. But then, a legitimate query arises here: Must $k=0,1,2 \ldots$ be left to run indefinitely (that is, from zero to infinity)? In other words: Is there any constraint on the velocity of the superluminal electron? For an answer to this question, see, for example, Stecker (2014) who studies and discusses the limits on superluminal electron and neutrino velocities.

\section{Conclusions}

The above calculations show, in the strict limit of realistic systems of single particle, using positive energy only and waves propagating only in the $\mathrm{z}$ - direction, that the expectation value of the relative linear velocity component of a spin- $1 / 2$ particle exceeds the speed of light. Under these restrictions, it can be noted that what so far appears to be a disaster (namely, the violation of causality by spin angular momentum) can prove to be a highly desirable aspect of spin-1/2 field theory. Finally, this work shows explicitly that the phenomenon of spin exists not only at low and relativistic velocities, but at superluminal propagation, as well.

\section{Acknowledgements}

Banini K. G. and Nuviadenu C.'s help with proofreading this work is appreciated.

\section{References}

Anton, H. (1987). Elementary Linear Algebra (5th ed.). Canada: Anton Textbooks.

Carmeli, M. (2000). Group Theory and General Relativity-Representations of the Lorentz Group and their Applications to the Gravitational Field. London: Imperial College Press.

Gelfand, I. M., \& Fomin, S. V. (1991). Calculus of Variations. New York, Mineola: Dover Publications, Inc.

Greiner, W. (1994). Quantum Mechanics-An Introduction (3rd ed.). Berlin, Heidelberg: Springer.

Greiner, W. (2000) . Relativistic Quantum Mechanics-Wave Equations (3rd ed.). Berlin, Heidelberg: Springer.

Greiner, W., \& Reinhardt, J. (1996). Field Quantization (2nd ed.). Berlin, Heidelberg: Springer.

Guidry, M. (1991). Gauge Field Theories-An Introduction with Applications. New York: John Wiley \& Sons, Inc.

Modanese, G. (2013, April 7). Velocity Requirements for Causality Violation. arXiv: 1304.5522 v 1, physics.gen-ph.

Naber, G. L. (1992). The Geometry of Minkowski Spacetime. New York: Springer-Verlag.

Ohanian, H. C. (1984, February 5). What Is Spin?. New York.

Shore, G. M. (2003, February 28). Causality and Superluminal Light. World Scientific: 0302116.

Stecker, F. W. (2014, February 12). Limiting Superluminal Electron and Neutrino Velocities using the 2010 Crab Nebula Flare and the IceCube PeV Neutrino Events. arXiv: 1306.6095v6 [hep-ph].

\section{Copyrights}

Copyright for this article is retained by the author(s), with first publication rights granted to the journal.

This is an open-access article distributed under the terms and conditions of the Creative Commons Attribution license (http://creativecommons.org/licenses/by/3.0/). 